\title{
Choroidal and Retinal Abnormalities by Optical Coherence Tomography in Endogenous Cushing's Syndrome
}

OPEN ACCESS

Edited by:

Pierrette Gaudreau,

Université de Montréal, Canada

Reviewed by: Gábor B. Makara,

Hungarian Academy of

Sciences, Hungary

Nils Lambrecht,

University of California Irvine, USA

${ }^{*}$ Correspondence:

Maria Cândida Villares

Barisson Fragoso maria.villares@hc.fm.usp.br

Specialty section:

This article was submitted to Neuroendocrine Science,

a section of the journal

Frontiers in Endocrinology

Received: 02 September 2016 Accepted: 28 November 2016 Published: 09 December 2016

Citation:

Abalem MF, Machado MC Santos HNVD, Garcia R, Helal J Jr., Carricondo PC, Pimentel SLG, Monteiro MLR, Qian CX, Bronstein MD and Fragoso MCVB (2016) Choroidal and Retinal Abnormalities by Optical Coherence

Tomography in Endogenous Cushing's Syndrome.

Front. Endocrinol. 7:154. doi: 10.3389/fendo.2016.00154
Maria Fernanda Abalem ${ }^{1,2}$, Marcio Carlos Machado ${ }^{3,4}$, Helen Nazareth Veloso Dos Santos ${ }^{1}$ Rafael Garcia1, John Helal Jr. ${ }^{1}$, Pedro Carlos Carricondo', Sérgio Luis Gianotti Pimentel', Mario Luiz Ribeiro Monteiro', Cynthia X. Qian', ${ }^{2,5}$ Marcello Delano Bronstein ${ }^{3}$ and Maria Cândida Villares Barisson Fragoso ${ }^{3 *}$

'Department of Ophthalmology and Otolaryngology, University of São Paulo Medical School, São Paulo, São Paulo, Brazil, ${ }^{2}$ Department of Ophthalmology and Visual Sciences, Kellogg Eye Center, University of Michigan, Ann Arbor, MI, USA, ${ }^{3}$ Department of Endocrinology, University of São Paulo Medical School, São Paulo, São Paulo, Brazil, ${ }^{4}$ Endocrinology Service, AC Camargo Cancer Center, São Paulo, São Paulo, Brazil, ${ }^{5}$ Department of Ophthalmology, MaisonneuveRosemont Hospital Research Centre, University of Montreal, Montreal, QC, Canada

Context: Cortisol has been suggested as a risk factor for choroidal thickening, which may lead to retinal changes.

Objective: To compare choroidal thickness measurements using optical coherence tomography (OCT) in patients with endogenous active Cushing's syndrome (CS) and to evaluate the occurrence of retinal abnormalities in the same group of patients.

Design: Cross-sectional study.

Setting: Outpatient clinic.

Patients: Eleven female patients with CS in hypercortisolism state as determined by the presence of at least two abnormal measurements from urinary cortisol $24 \mathrm{~h}$, no suppression of cortisol with low dose dexamethasone suppression test, and nocturnal salivary cortisol levels and 12 healthy controls.

Methods: Choroidal and retinal morphology was assessed using OCT.

Main outcome measures: Choroidal thickness measurements and the presence of retinal changes.

Results: The mean subfoveal choroidal thickness was $372.96 \pm 73.14 \mu \mathrm{m}$ in the patients with CS and $255.63 \pm 50.70 \mu \mathrm{m}$ in the control group $(p<0.001)$. One patient (9.09\%) presented with central serous chorioretinopathy and one patient (9.09\%) with pachychoroid pigment epitheliopathy.

Conclusion: Choroidal thickness is increased in the eyes of patients with active CS compared to healthy and matched control. Also, 18.18\% of patients presented with macular changes, possibly secondary to choroidal thickening. While further studies are necessary to confirm our findings, excess corticosteroid levels seem to have a significant effect on the choroid and might be associated with secondary retinal diseases.

Keywords: hypercortisolism, Cushing syndrome, choroid, optical coherence tomography, eye 


\section{INTRODUCTION}

Endogenous Cushing's syndrome (CS) is a prototype of metabolic syndrome due to chronic exposure to excess cortisol. The clinical presentation varies depending mostly on the time span the patient is submitted to excessive cortisol production and is characterized by weight gain, facial plethora, central obesity, proximal muscle weakness, hypertension, diabetes mellitus, osteoporosis, neuropsychological disturbances, and purple striae (1).

Several previous reports have documented visual loss in endogenous CS resulting from central serous chorioretinopathy (CSC), a disease that impairs central vision and is characterized by serous retinal detachment, retinal pigment epithelium (RPE) changes, and choroidal vascular hyperpermeability (2-4). While the pathogenesis of CSC is still poorly understood, several risk factors seem to be involved such as younger age, male gender, type-A behavioral pattern, history of organ transplant, pregnancy, systemic blood hypertension, use of exogenous corticosteroids, and endogenous hypercortisolism (5-7).

The mechanisms by which corticosteroids are implicated in the development of CSC are unclear. Currently, it is believed that cortisol excess may lead to a number of choroid abnormalities including (a) increased capillary fragility, leading to choroidal circulation imbalance and leakage of fluid to the subretinal space; (b) blood coagulation in the choroid, leading to choroidal hypoperfusion; (c) inhibition of collagen formation affecting the main component of Bruch's membrane, the innermost choroid layer; (d) impact on ion and water transport of epithelial cells through interference on mineralocorticoid receptors; and (e) induce systemic hypertension, which in itself is a risk factor for CSC (7).

The choroid is primarily a vascular tissue, which provides the major blood supply to the retina. It can be assessed by two different methods: indocyanine green angiography (ICG), which is a somewhat invasive method, and by enhanced depth image optical coherence tomography (EDI-OCT), a non-invasive exam. Patients with CSC often present choroid vascular hyperpermeability on ICG and increased choroidal thickness when measured by EDI-OCT (8-11).

Several reports have reported increased choroidal thickness in both affected and fellow eyes of CSC patients, compared to control subjects. Moreover, in cases of unilateral CSC, the affected eye seems to be thicker than the fellow eye (12-14).

Because of the previous association of CSC both with excess corticosteroid levels and choroidal thickening, we hypothesized that patients with endogenous hypercortisolism might develop choroidal thickening that could be a predisposing factor for CSC and other diseases possibly associated to choroidal thickening such as pachychoroid pigment epitheliopathy (PPE), pachychoroid neovasculopathy (PN), and polypoidal choroidal vasculopathy (PCV) $(15,16)$.

More recently, Karaca et al. reported a thicker choroid in patients with CS possibly related to increased levels of ACTH itself, increased levels of plasma cortisol, or both (17). Therefore, the aim of the present study was to evaluate possible effects of excess corticosteroids exposure on choroidal thickness using OCT and to investigate possible retinal abnormalities in patients with active hypercortisolism due to endogenous CS.

\section{MATERIALS AND METHODS}

This cross-sectional study was performed at the Division of Ophthalmology and the Department of Endocrinology of the University of São Paulo Medical School. The study was approved by our institutional review board and conducted according to the Declaration of Helsinki. Informed written consent was obtained from all participants.

A diagnosis of active CS was established by the presence of at least two unequivocal altered of first line screening for CS [urinary cortisol (UC) $24 \mathrm{~h}$, nocturnal serum, or salivary cortisol] and serum cortisol (Fs) after low dose dexamethasone suppression test ( $F \mathrm{~s}>1.8 \mu \mathrm{g} / \mathrm{dL}$ or $50 \mathrm{mmol} / \mathrm{L}$ ). Eleven patients were selected for the study, nine due to ACTH-dependent and two due to ACTH-independent CS. Twelve healthy patients were recruited as a control group, matched for age and gender to the patients. Control patients with systemic diseases, like arterial systemic hypertension, diabetes, autoimmune diseases, or any other condition that could affect the choroid and retina were excluded. Moreover, those undertaking exogenous steroids in either anti-inflammatory or immunosuppressive dose in the past 12 months were also excluded.

Considering that CSC can be present unilaterally, both eyes were included. Exclusion criterion for patients were the presence of refractive error exceeding \pm 6.00 diopters (D) of spherical equivalent, presence of more than two diopters of keratometric astigmatism, axial length of more than $26.5 \mathrm{~mm}$, important media opacity (resulting in poor OCT image quality), history of uveitis and retinal diseases other than CSC, and any choroidal-related diseases, such as PPE, PN, and PCV. Patients with a history of glaucoma or any other optic neuropathy, intraocular surgery in the past 3 months (including cataract surgery), intravitreal injections (steroids and/or anti-vascular endothelial growth factor), and/or laser treatment were also excluded.

\section{Patient Assessment}

All patients were submitted to a comprehensive ocular examinations, including biomicroscopy of anterior and posterior segments, indirect fundoscopy, axial length measurement (IOL Master, Carl Zeiss, Jena, Germany), and spectral-domain optical coherence tomography (SD-OCT) (Spectralis; Heidelberg Engineering, Heidelberg, Germany) with an EDI protocol (horizontal and vertical scans, 7 sections, high resolution mode, 25 frames). SD-OCT images were routinely obtained at the same time, avoiding diurnal variations of choroidal vasculature. Two raters experienced in examining EDI-OCT images (Maria Fernanda Abalem and Helen Nazareth Veloso Dos Santos) independently evaluated the choroidal features. The third rater (Pedro Carlos Carricondo) was consulted when the two raters disagreed.

\section{Choroidal Evaluation}

The following features were addressed, as described previously $(10,18)$ :

(A) Total choroidal thickness (TCT) was measured at the subfoveal position. The measures were performed manually using the caliper of the Eye Explorer software (v. 6.0.9.0; 
Heidelberg Engineering) with $80 \%$ zoom. The measure was obtained perpendicularly, from the outer edge of the hyperreflective RPE to the hyperreflective inner sclera.

(B) Choroidal segmentation: three large choroidal vessels measuring at least $100 \mu$ in diameter, located subfoveal, and a perpendicular line from the innermost point of each one was drawn so these lines would intersect with the lines used to measure TCT. The Haller layer was determined by measuring perpendicularly from the inner border of the sclera to the innermost line of the selected large choroidal vessels. The remaining distance of choroidal thickness was considered as the choriocapillaris/Sattler layer complex. These two layers were analyzed together because current OCT technology still does not allow for the separation of both layers.

\section{Retinal Evaluation}

The main characteristics of CSC, PPE, PN, and PCV were addressed, including the presence of subretinal fluid, intraretinal cysts, ellipsoid layer disruption, drusen, pigmented epithelium detachment, and choroidal neovascularization.

\section{Statistical Analysis}

All statistical analyses were performed using the SPSS software version 18.0 (SPSS, Chicago, IL, USA). Numerical values were reported as mean $\pm \mathrm{SD}$. Choroidal thickness parameters, including the thickness of Haller layer, the choriocapillaris/Sattler layer were estimated and compared. The interobserver reliability was expressed as the intraclass correlation coefficient. Also, $p$ values of 0.05 were considered statistically significant.

Optical coherence tomography measurements of both eyes were compared by using Generalized Estimating Equation (GEE) models, in order to compensate inter-eye dependencies. In this study, all patients and controls had both eyes included. As eyes of the same individual were expected to have some degree of intercorrelation with respect to OCT parameters, GEE models were used to adjust for within-patient inter-eye correlations. GEE models are generalized linear models that allow for the specification of within-group correlations when evaluating the ability of one or several independent variables to predict a dependent variable.

\section{RESULTS}

From 2010 to 2015 , a total of 11 patients with active endogenous CS, mean age of 38.45 years $( \pm 16.32)$, and 12 control patients, mean age of 51.33 years $( \pm 16.64)$, were included in this study. By chance, all of them were female subjects. There was no statistical difference between age of patients and controls $(p=0.08)$. The etiologies of CS were eight patients with (72.7\%) Cushing's disease (pituitary adenoma), one (9.1\%) with adrenocotical adenoma, one $(9.1 \%)$ with adrenocortical carcinoma, and one (9.1\%) with primary macronodular adrenal hyperplasia (PMAH). None of our patients had pituitary tumor with suprasellar extension as confirmed by magnetic resonance imaging. As required to be eligible, all patients were in hypercortisolism state according to UC $24 \mathrm{~h}$ levels and nocturnal salivary cortisol (patient number 9) (Table 1). No control patient presented with any autoimmune and/or vascular systemic and ocular condition as required to be included in this study. However, one patient had prior history of breast cancer diagnosed and surgically treated 10 years ago, and two patients had prior history of cholecystectomy in the past 5 years due to chronic cholecystitis. Any patient had history of exogenous steroids intake in the past 12 months (Table 2).

The interobserver reliability of the two retinal specialists who performed the measurements was very good. The intraclass correlation coefficients were of 0.876 for TCT, 0.864 for the thickness of Haller layer, and 0.996 for the choriocapillaris/Sattler layer.

The mean subfoveal choroidal thickness was $372.96 \pm 73.14 \mu \mathrm{m}$ in eyes of patients with CS and $255.63 \pm 50.70 \mu \mathrm{m}$ in eyes of the control group, with statistically significant difference $(p<0.001$, GEE) (Figure 1). The results were similar in the mean thickness

\begin{tabular}{|c|c|c|c|c|c|c|c|c|}
\hline$N$ & Patient & $\begin{array}{c}\text { Age } \\
\text { (years old) }\end{array}$ & Gender & $\begin{array}{l}\text { UC } 24 \mathrm{~h} \\
(\mu \mathrm{g} / 24 \mathrm{~h})\end{array}$ & $\begin{array}{c}\text { ACTH } \\
(\mathrm{pg} / \mathrm{mL})\end{array}$ & Etiology & $\begin{array}{l}\text { Pituitary } \\
\text { MRI }\end{array}$ & $\begin{array}{l}\text { Active hypercortisolism } \\
\text { state (months) }\end{array}$ \\
\hline 1 & APS & 33 & Female & $837^{\mathrm{a}}$ & 126.2 & $C D$ & MICRO & 115 \\
\hline 2 & DPM & 22 & Female & $502^{a}$ & 42.5 & CD & MACRO & 28 \\
\hline 3 & FSF & 32 & Female & $399^{b}$ & 126.8 & CD & MACRO & 90 \\
\hline 4 & LLA & 35 & Female & $1288^{a}$ & 42.2 & $C D$ & MICRO & 5 \\
\hline 5 & MLP & 51 & Female & $826^{a}$ & 42.2 & $\mathrm{CD}$ & MACRO & 38 \\
\hline 6 & MISO & 45 & Female & $1485^{a}$ & 87.1 & $C D$ & MICRO & 15 \\
\hline 7 & PRM & 29 & Female & $371^{a}$ & 33.2 & CD & MICRO & 80 \\
\hline 8 & SAGP & 26 & Female & $334^{b}$ & 77.5 & CD & MACRO & 18 \\
\hline 9 & VAS & 69 & Female & $295^{a} / 0.8^{c}$ & $<2.0$ & $\begin{array}{l}\mathrm{CS} \\
\mathrm{PMAH}\end{array}$ & - & 360 \\
\hline 10 & GMS & 19 & Female & $982^{a}$ & $<2.0$ & $\begin{array}{l}\text { CS } \\
\text { Adenoma }\end{array}$ & - & 48 \\
\hline 11 & VLCL & 62 & Female & $733^{a}$ & $<2.0$ & $\begin{array}{l}\text { CS } \\
\text { Carcinoma }\end{array}$ & - & 12 \\
\hline
\end{tabular}

UC, urinary cortisol $24 \mathrm{~h}$ (normal range: a Reference: 50-310 $\mu \mathrm{g} / 24 \mathrm{~h}$ or ${ }^{\mathrm{b}}$ Reference: 3-43 $\mu \mathrm{g} / 24 \mathrm{~h}$ ); 'Midnight salivary cortisol (normal value: up to 0.12 $\mu \mathrm{g} / \mathrm{dL}$ ); ACTH (normal range: 7.2-63.30 pg/mL); CD, Cushing's disease; CS, Cushing's syndrome; PMAH, primary macronodular adrenal hyperplasia; MRI, magnetic resonance imaging; MICRO (microadenoma $<10 \mathrm{~mm}$ ); MACRO (macroadenoma $\geq 10 \mathrm{~mm}$ ) based on MRI. 
TABLE 2 | Demographical and clinical data of control patients.

\begin{tabular}{rlcll}
\hline $\boldsymbol{N}$ & Patient & Age (years) & Gender & Comorbidities \\
\hline 1 & MEGB & 27 & Female & No \\
2 & APP & 27 & Female & No \\
3 & MS & 29 & Female & No \\
4 & CSS & 43 & Female & No \\
5 & NAO & 51 & Female & No \\
6 & ESL & 54 & Female & Cholescystitis \\
7 & DMC & 55 & Female & Breast cancer \\
8 & HHR & 60 & Female & No \\
9 & MHGK & 60 & Female & Cholescystitis \\
10 & ESG & 65 & Female & No \\
11 & VB & 68 & Female & No \\
12 & ANB & 77 & Female & No
\end{tabular}

asurgically treated.

of Haller layer, measuring $342.05 \pm 73.77 \mu \mathrm{m}$ in eyes of patients with CS and $231.92 \pm 51.54 \mu \mathrm{m}$ in eyes of the control group $(p<0.001, \mathrm{GEE})$. The mean thickness of choriocapillaris/Sattler layer also was statistically different, measuring $34.91 \pm 22.36 \mu \mathrm{m}$ in the group with CS and $23.71 \pm 14.93 \mu \mathrm{m}$ in the control group $(p<0.001, \mathrm{GEE})$.

Two patients (18.18\%) presented with macular changes. Patient 1 presented drusen-like lesions, associated with ellipsoid zone irregularity on both eyes, suggesting PPE. Patient 10 presented with bilateral subretinal fluid, associated to ellipsoid zone irregularity and drusen-like lesions, suggestive of CSC (Figure 2).

\section{DISCUSSION}

Cushing's syndrome is a medical condition that can be associated with numerous deleterious changes in various organ systems leading to comorbidities and increased mortality ratio. This study addressed possible effects of excessive corticosteroid levels on the choroid in patients with active hypercortisolism due to endogenous CS. We have found that TCT was significantly greater in patients with endogenous CS, when compared with age- and gender-matched controls, without any history of systemic and ocular vascular or autoimmune condition that could directly or indirectly affect both retina and choroid as well as prior history of exogenous steroids intake in the past 12 months.

Our findings are in agreement with a number of previous studies, which demonstrated a thicker choroid in patients with CSC, a condition that is also related to elevated systemic levels of corticosteroids. Several reports identified corticosteroids as a significant risk factor for the development of acute, exudative macular manifestation in patients with CSC, either by endogenous Cushing syndrome or by exogenous intake (2, 7, 19, 20). Garg et al. also demonstrated an increase in the 8:00 a.m. and 11:00 p.m. serum cortisol and UC $24 \mathrm{~h}$ levels in acute CSC patients compared to healthy subjects $(21,22)$.

Additionally, other endocrine abnormalities were found in a case series of 24 patients with CSC, such as UC $24 \mathrm{~h}$ and tetrahydroaldosterone levels, low serum aldosterone levels, elevated single morning plasma catecholamine level, but normal 24-h urine metanephrines (23). The current study is partially in accordance with a recent study by Karaca et al. that encountered an association between choroidal thickening and ACTH levels in patients with chronic CS, suggesting that ACTH itself, increased cortisol levels, or both might be related to this finding (17). However, in this study it is not clear about the cortisol state of patients because only the midnight and morning serum cortisol were evaluated. It is possible that these patients had only absence of circadian secretion due to medical treatment (e.g., cabergoline, ketoconazole, or pasireotide) in the presence of normal levels of UC $24 \mathrm{~h}$ (24). The total cortisol secretion measured by UC $24 \mathrm{~h}$ should have been demonstrated to characterize hypercortisolism activity. Moreover, to the best of our knowledge, there are no melanocortin receptors on the choroid; hence, it seems more likely that increased cortisol levels may be responsible for the choroidal changes.

The choroid can change their volume, thereby their thickness as much as fourfold over a few days. There are five possible mechanisms underlying choroidal thickening. The most likely mechanism is due to the synthesis of osmotically active molecules that act as "sponges" leading to expansion of the lacunae (25). Another mechanism refers to changes in vascular permeability. An increase in capillary permeability may allow proteins to move into the extracellular matrix and/or lymphatic, followed by passive fluid flow (26). A third mechanism proposed derives from the aqueous humor flow from the anterior chamber to the choroid. Since the choroid is part of the uveoscleral outflow pathway, an increase in the humor aqueous flow may be transmitted to the choroid and expand it $(25,27)$. Another similar mechanism is by moving fluid across the RPE. There is a flow of ions and water between the retina and the choroid, and changes in this flow might impact on choroidal thickness (28). Finally, the choroid may thicken because of changes in the tonus of non-vascular smooth muscle cells that are controlled by both sympathetic and parasympathetic inputs. If these cells relax, the choroid may become thicker (29-31).

The excess of cortisol has several effects that may be involved in the mechanisms that modulate choroidal thickness contributing for both thickening and thinning. Corticosteroids may increase osmolarity and active fluid flow into the choroidal vasculature (32); increase renal tubular sodium reabsorption and intravascular volume expansion (33); dilate choroidal endothelial cells $(33,34)$; and inhibit the formation of collagen, which may facilitate moving fluid across the RPE (7). On the other side, corticosteroids impair vasodilation, induce vasoconstriction (also by enhancement of the renin angiotensin system), and increase the vascular sensitivity to the effects of catecholamines (33). These effects are thought to thin the choroid. We believe the thickening mechanisms have predominated because thinning is somewhat limited by mechanical restrictions and because intravascular expansion may lead to expansion of the lacunae, which is the major anatomical change, related to choroidal thickening (31).

In the current study, the Haller layer thickness was greater in patients with CS than in controls. The choriocapillaris/Sattler layer complex did not show any difference in both groups. These findings suggest that increased TCT was at least in part due to increased Haller layer thickness. Chung et al. also assessed the Haller and Sattler layers in patients with CSC and showed a 

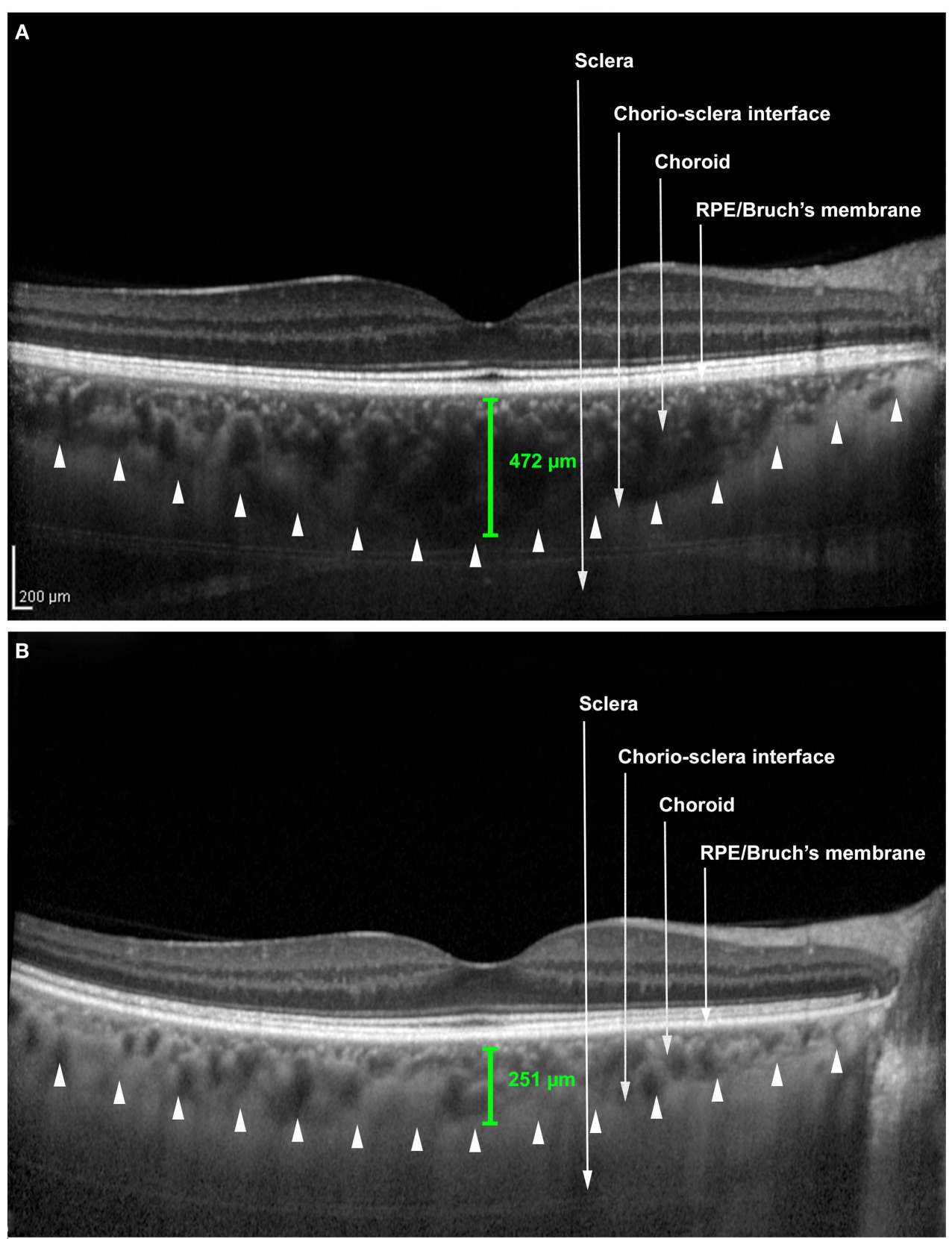

FIGURE 1 | Enhanced depth image optical coherence tomography scans. (A) Patient with Cushing's syndrome. (B) Control patient. The caliper (green) represents the choroidal thickness. The arrowheads represent the anterior boundary of the sclera.

greater ratio of Haller layer/TCT. They believed these findings were possibly due to the higher number of non-vascular smooth muscle cells in the Haller layer, which may stretch out, leading to vascular dilation and accumulation of fluid in the stroma. Alternatively, the lower number of these cells in the choriocapillaris/Sattler layers might keep these layers relatively stable under sympathetic stimuli (10). Furthermore, the lacunae are more predominant and larger in the suprachoroid, which may explain a greater vascular expansion in this location (31).
Han et al. prospectively evaluate the effects of corticosteroids on choroidal thickness in patients who required high-dose corticosteroid pulse therapy for a short period, with a 1-month follow-up (35). While the authors did not find changes in choroidal thickness, they described some features of CSC like pigment epithelial detachment and subretinal fluid in one patient who also presented with a thicker choroid. This lack of consistent changes might be explained by the fact that these patients were under high levels of cortisol, but for a short period. In our study, all 

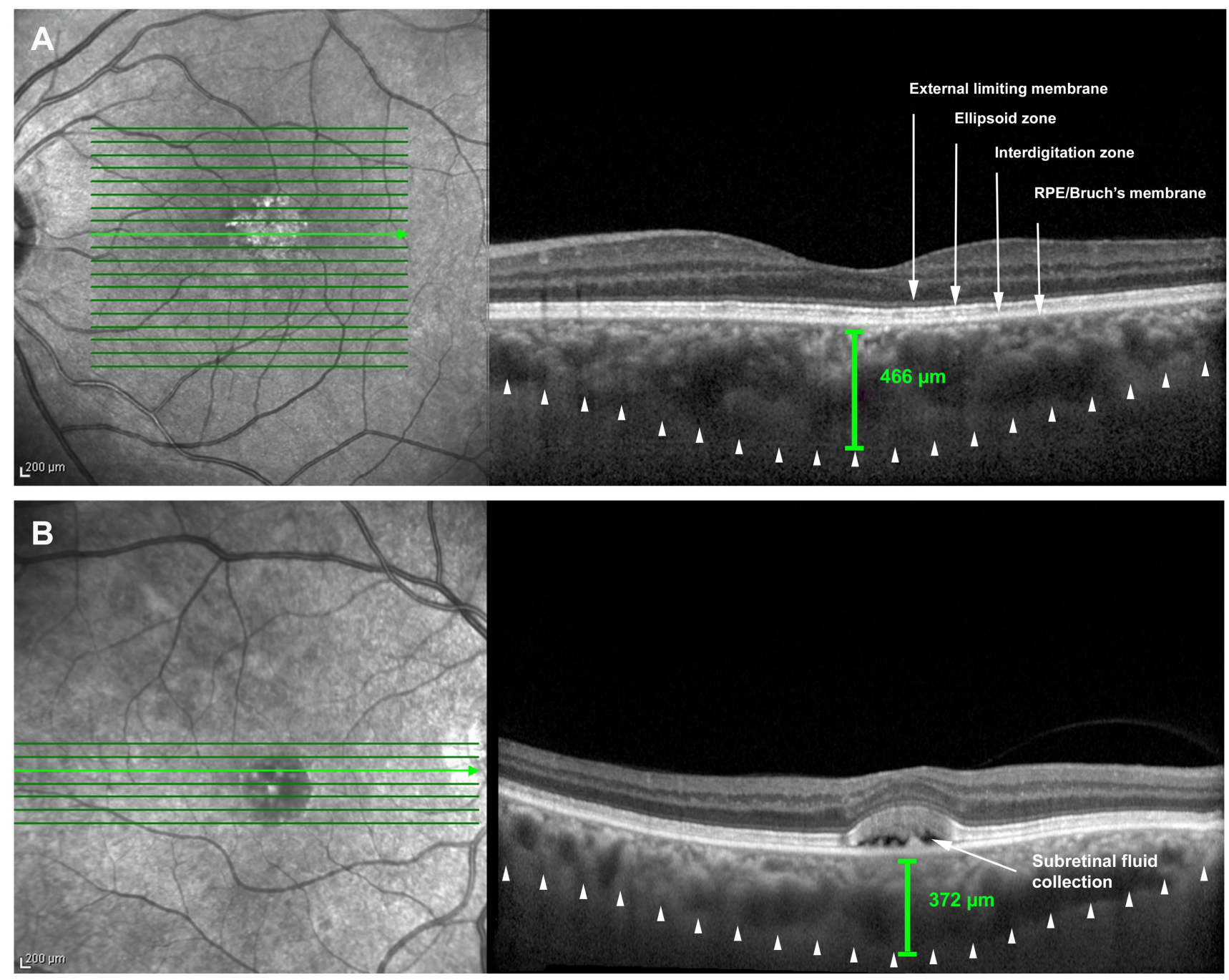

FIGURE 2 Macular optical coherence tomography scans. (A) Left eye of patient 1 with drusen-like lesions, associated with ellipsoid zone irregularity and choroidal thickening. (B) Right eye of patient 10 with subretinal fluid (arrow), associated to ellipsoid zone irregularity and choroidal thickening.

patients were under prolonged and excessive cortisol exposure, suggesting that choroidal thickening also depend on both factors: degree and duration of hypercortisolism, as occurs with systemic manifestations (35).

We also found one patient (9.09\%) with a PPE and another (9.09\%) with bilateral CSC, conditions that were demonstrated to be associated with choroidal thickening $(10,15)$. The frequency of PPE in overall population is still unknown. It is a misdiagnosed condition, in which there is a marked disturbance in the RPE cells with possible visual impairment (15). According to Bouzas et al., the incidence of CSC is $5 \%$ in patients with endogenous CS. In this study, 3 out of 46 women and 1 out of 14 men were affected (2). We believe this condition might be underestimated in patients with CS, because (1) during the hypercortisolism state, the patient and the physician might not note this kind of symptom and (2) CSC is self-limited in most cases and visual acuity usually returns to $20 / 25$ or better $(36,37)$, although chronic and atypical cases may also occur in $30-50 \%$ of cases $(7,38,39)$. Thus, we believe patients with CS might require proper retinal evaluation.

To our knowledge, this is the first study to describe choroidal and retinal abnormalities in patients with active endogenous CS-dependent and independent of ACTH during hypercortisolism state, based on high levels of UC $24 \mathrm{~h}$, except in the patient with PMAH, where cortisol activity was characterized based on both midnight salivary cortisol and low dose dexamethasone suppression test. As increased cortisol activity is the hallmark of CS, this specific group of patients provides better understanding of the effects of cortisol on the choroid. We believe the increased osmolarity, the mineralocorticoid effects of cortisol, and the activation of the renin angiotensin system in patients with CS may be involved in the pathophysiology of choroidal expansion explaining the increased choroidal thickness measurements.

The current study has a number of limitations including the cross-sectional design and the small number of patients. 
However, our data serve to emphasize the existence of an additional morbidity in patients with endogenous CS. Further comparative studies are therefore required to better evaluate (1) the role of other adrenal hormones, such as catecholamines that also seem to be involved in the pathogenesis of CSC (40, 41); (2) the long-term effects of cortisol on both the choroid and the retina of these patients; and (3) the visual impairment caused by such abnormalities. Since choroidal thickening is associated to retinal conditions, like CSC, PPE, PN, and PCV, patients with CS in hypercortisolism state should be evaluated for these conditions.

\section{ETHICS STATEMENT}

Comissão de Ética para Análise de Projetos de Pesquisa do Hospital das Clinicas da Faculdade de Medicina da Universidade

\section{REFERENCES}

1. Nieman LK. Cushing's syndrome: update on signs, symptoms and biochemical screening. Eur J Endocrinol (2015) 173(4):M33-8. doi:10.1530/EJE15-0464

2. Bouzas EA, Scott MH, Mastorakos G, Chrousos GP, Kaiser-Kupfer MI. Central serous chorioretinopathy in endogenous hypercortisolism. Arch Ophthalmol (1993) 111(9):1229-33. doi:10.1001/archopht.1993.010900900 81024

3. Giovansili I, Belange G, Affortit A. Cushing disease revealed by bilateral atypical central serous chorioretinopathy: case report. Endocr Pract (2013) 19(5):e129-33. doi:10.4158/EP12389.CR

4. Iannetti L, Spinucci G, Pesci FR, Vicinanza R, Stigliano A, Pivetti-Pezzi P. Central serous chorioretinopathy as a presenting symptom of endogenous Cushing syndrome: a case report. Eur JOphthalmol (2011) 21(5):661-4. doi:10.5301/EJO.2011.6449

5. Abalem MF, Carricondo PC, Pimentel SL, Takahashi WY. Idiopathic organ transplant chorioretinopathy after liver transplantation. Case Rep Ophthalmol Med (2015) 2015:964603. doi:10.1155/2015/964603

6. Daruich A, Matet A, Dirani A, Bousquet E, Zhao M, Farman N, et al. Central serous chorioretinopathy: recent findings and new physiopathology hypothesis. Prog Retin Eye Res (2015) 48:82-118. doi:10.1016/j.preteyeres. 2015.05.003

7. Bouzas EA, Karadimas P, Pournaras CJ. Central serous chorioretinopathy and glucocorticoids. Surv Ophthalmol (2002) 47(5):431-48. doi:10.1016/ S0039-6257(02)00338-7

8. Pang CE, Shah VP, Sarraf D, Freund KB. Ultra-widefield imaging with autofluorescence and indocyanine green angiography in central serous chorioretinopathy. Am J Ophthalmol (2014) 158(2):362.e-71.e. doi:10.1016/j. ajo.2014.04.021

9. Hirahara S, Yasukawa T, Kominami A, Nozaki M, Ogura Y. Densitometry of choroidal vessels in eyes with and without central serous chorioretinopathy by wide-field indocyanine green angiography. Am J Ophthalmol (2016) 166:103-11. doi:10.1016/j.ajo.2016.03.040

10. Chung YR, Kim JW, Kim SW, Lee K. Choroidal thickness in patients with central serous chorioretinopathy: assessment of Haller and Sattler layers. Retina (2016) 36(9):1652-7. doi:10.1097/IAE.0000000000000998

11. Lee H, Bae K, Kang SW, Woo SJ, Ryoo NK, Kim SJ, et al. Morphologic characteristics of choroid in the major choroidal thickening diseases, studied by optical coherence tomography. PLoS One (2016) 11(1):e0147139. doi:10.1371/ journal.pone.0147139

12. Imamura Y, Fujiwara T, Margolis R, Spaide RF. Enhanced depth imaging optical coherence tomography of the choroid in central serous chorioretinopathy. Retina (2009) 29(10):1469-73. doi:10.1097/IAE.0b013e3181be0a83

13. Jirarattanasopa P, Ooto S, Tsujikawa A, Yamashiro K, Hangai M, Hirata $\mathrm{M}$, et al. Assessment of macular choroidal thickness by optical coherence de São Paulo. This is a non-interventional study. The patients underwent routine ophthalmic evaluation and optical coherence tomography. The latter does not require any additional procedure and/or preparing. It is a non-invasive imaging method. The patients were told about the minimum risk of participating in this study and also about the possibility of being treated in case of eye-disease finding.

\section{AUTHOR CONTRIBUTIONS}

MA: design, data collection, data analysis, and writing; MCM: design, data analysis, and reviewing; HS and RG: data collection; PC: design, statistical analysis, data analysis, and reviewing; $\mathrm{JH}$ : design, data analysis, and reviewing; SP: reviewing; CQ: data collection and figures edition; MB: design and reviewing; MF: design, data collection, data analysis, and reviewing.

tomography and angiographic changes in central serous chorioretinopathy. Ophthalmology (2012) 119(8):1666-78. doi:10.1016/j.ophtha.2012.02.021

14. Maruko I, Iida T, Sugano Y, Ojima A, Sekiryu T. Subfoveal choroidal thickness in fellow eyes of patients with central serous chorioretinopathy. Retina (2011) 31(8):1603-8. doi:10.1097/IAE.0b013e31820f4b39

15. Warrow DJ, Hoang QV, Freund KB. Pachychoroid pigment epitheliopathy. Retina (2013) 33(8):1659-72. doi:10.1097/IAE.0b013e3182953df4

16. Pang CE, Freund KB. Pachychoroid neovasculopathy. Retina (2015) 35(1):1-9. doi:10.1097/IAE.0000000000000331

17. Karaca C, Karaca Z, Kahraman N, Sirakaya E, Oner A, Mirza GE. Is there a role of ACTH in increased choroidal thickness in Cushing syndrome? Retina (2016). doi:10.1097/IAE.0000000000001198

18. Branchini LA, Adhi M, Regatieri CV, Nandakumar N, Liu JJ, Laver N, et al. Analysis of choroidal morphologic features and vasculature in healthy eyes using spectral-domain optical coherence tomography. Ophthalmology (2013) 120(9):1901-8. doi:10.1016/j.ophtha.2013.01.066

19. Carvalho-Recchia CA, Yannuzzi LA, Negrao S, Spaide RF, Freund KB, Rodriguez-Coleman $\mathrm{H}$, et al. Corticosteroids and central serous chorioretinopathy. Ophthalmology (2002) 109(10):1834-7. doi:10.1016/S01616420(02)01117-X

20. Harada T, Harada K. Six cases of central serous choroidopathy induced by systemic corticosteroid therapy. Doc Ophthalmol (1985) 60(1):37-44. doi:10.1007/BF00164568

21. Zakir SM, Shukla M, Simi ZU, Ahmad J, Sajid M. Serum cortisol and testosterone levels in idiopathic central serous chorioretinopathy. Indian J Ophthalmol (2009) 57(6):419-22. doi:10.4103/0301-4738.57143

22. Garg SP, Dada T, Talwar D, Biswas NR. Endogenous cortisol profile in patients with central serous chorioretinopathy. Br J Ophthalmol (1997) 81(11):962-4. doi:10.1136/bjo.81.11.962

23. Haimovici R, Rumelt S, Melby J. Endocrine abnormalities in patients with central serous chorioretinopathy. Ophthalmology (2003) 110(4):698-703. doi:10.1016/S0161-6420(02)01975-9

24. Feelders RA, Hofland LJ. Medical treatment of Cushing's disease. JClin Endocrinol Metab (2013) 98(2):425-38. doi:10.1210/jc.2012-3126

25. Wallman J, Wildsoet C, Xu A, Gottlieb MD, Nickla DL, Marran L, et al. Moving the retina: choroidal modulation of refractive state. Vision Res (1995) 35(1):37-50. doi:10.1016/0042-6989(94)E0049-Q

26. Rada JA, Palmer L. Choroidal regulation of scleral glycosaminoglycan synthesis during recovery from induced myopia. Invest Ophthalmol Vis Sci (2007) 48(7):2957-66. doi:10.1167/iovs.06-1051

27. Pendrak K, Papastergiou GI, Lin T, Laties AM, Stone RA. Choroidal vascular permeability in visually regulated eye growth. Exp Eye Res (2000) 70(5):629-37. doi:10.1006/exer.2000.0825

28. Rymer J, Wildsoet CF. The role of the retinal pigment epithelium in eye growth regulation and myopia: a review. Vis Neurosci (2005) 22(3):251-61. doi:10.1017/S0952523805223015 
29. Kee CS, Hung LF, Qiao-Grider Y, Ramamirtham R, Winawer J, Wallman J, et al. Temporal constraints on experimental emmetropization in infant monkeys. Invest Ophthalmol Vis Sci (2007) 48(3):957-62. doi:10.1167/ iovs.06-0743

30. Stubinger K, Brehmer A, Neuhuber WL, Reitsamer H, Nickla D, Schrodl F. Intrinsic choroidal neurons in the chicken eye: chemical coding and synaptic input. Histochem Cell Biol (2010) 134(2):145-57. doi:10.1007/ s00418-010-0723-9

31. Nickla DL, Wallman J. The multifunctional choroid. Prog Retin Eye Res (2010) 29(2):144-68. doi:10.1016/j.preteyeres.2009.12.002

32. Oray M, Abu Samra K, Ebrahimiadib N, Meese H, Foster CS. Long-term side effects of glucocorticoids. Expert Opin Drug Saf (2016) 15(4):457-65. doi:10. $1517 / 14740338.2016 .1140743$

33. Sharma ST, Nieman LK. Cushing's syndrome: all variants, detection, and treatment. Endocrinol Metab Clin North Am (2011) 40(2):379-91, viii-ix. doi:10.1016/j.ecl.2011.01.006

34. Zhao M, Celerier I, Bousquet E, Jeanny JC, Jonet L, Savoldelli M, et al. Mineralocorticoid receptor is involved in rat and human ocular chorioretinopathy. J Clin Invest (2012) 122(7):2672-9. doi:10.1172/JCI61427

35. Han JM, Hwang JM, Kim JS, Park KH, Woo SJ. Changes in choroidal thickness after systemic administration of high-dose corticosteroids: a pilot study. Invest Ophthalmol Vis Sci (2014) 55(1):440-5. doi:10.1167/iovs.13-12854

36. Klein ML, Van Buskirk EM, Friedman E, Gragoudas E, Chandra S. Experience with nontreatment of central serous choroidopathy. Arch Ophthalmol (1974) 91(4):247-50. doi:10.1001/archopht.1974.03900060257001

37. Folk JC, Thompson HS, Han DP, Brown CK. Visual function abnormalities in central serous retinopathy. Arch Ophthalmol (1984) 102(9):1299-302. doi:10.1001/archopht.1984.01040031049021
38. Castro-Correia J, Coutinho MF, Rosas V, Maia J. Long-term follow-up of central serous retinopathy in 150 patients. Doc Ophthalmol (1992) 81(4):379-86. doi:10.1007/BF00169099

39. Gilbert CM, Owens SL, Smith PD, Fine SL. Long-term follow-up of central serous chorioretinopathy. Br J Ophthalmol (1984) 68(11):815-20. doi:10.1136/ bjo.68.11.815

40. Balacco-Gabrieli C, Asciano F, Reibaldi A, Santoro M. [Central serous retinopathy. Etiopathogenetic and clinical considerations (author's transl)]. Ophthalmologica (1980) 181(5):251-60. doi:10.1159/000309062

41. Sun J, Tan J, Wang Z, Yang H, Zhu X, Li L. Effect of catecholamine on central serous chorioretinopathy. J Huazhong Univ Sci Technolog Med Sci (2003) 23(3):313-6. doi:10.1007/BF02829525

Conflict of Interest Statement: The authors declare that the research was conducted in the absence of any commercial or financial relationships that could be construed as a potential conflict of interest.

The handling Editor declared a shared affiliation, though no other collaboration, with the author CQ and states that the process nevertheless met the standards of a fair and objective review.

Copyright (C) 2016 Abalem, Machado, Santos, Garcia, Helal, Carricondo, Pimentel, Monteiro, Qian, Bronstein and Fragoso. This is an open-access article distributed under the terms of the Creative Commons Attribution License (CC BY). The use, distribution or reproduction in other forums is permitted, provided the original author(s) or licensor are credited and that the original publication in this journal is cited, in accordance with accepted academic practice. No use, distribution or reproduction is permitted which does not comply with these terms. 